\title{
A 13-Year Old Female with Pancytopenia at the Presentation of a Borrelia Hispanica Infection: Case Report and Review of the Literature
}

Irmin Leen ${ }^{1,2}$, Peggy Bruynseels ${ }^{3}$, Mark van Oort ${ }^{1}$ and Machiel van den Akker ${ }^{1,4^{*}}$

${ }^{1}$ Department of Pediatrics, Queen Paola Children's Hospital, Antwerp, Belgium

${ }^{2}$ Department of Emergency Medicine, ZNA Middelheim, Antwerp, Belgium

${ }^{3}$ Department of Microbiology, ZNA Middelheim, Antwerp, Belgium

${ }^{4}$ Department of Pediatric Hematology Oncology, UZ Brussel, Brussels, Belgium

"Corresponding author: Machiel van den Akker, Department of Pediatrics, Queen Paola Children's Hospital, Lindendreef 1, 2020 Antwerp, Belgium, Tel: 00316 81387488; Fax: 00323 2802133; Email: machielvdakker@gmail.com

Rec date: Aug 23, 2016; Acc date: Oct 04, 2016; Pub date: Oct 06, 2016

Copyright: (c) 2016 Leen I, et al. This is an open-access article distributed under the terms of the Creative Commons Attribution License, which permits unrestricted use, distribution, and reproduction in any medium, provided the original author and source are credited.

\begin{abstract}
Background: It is not uncommon that a child with a febrile illness of unknown etiology is admitted to the hospital. When the complete blood count reveals a pancytopenia, the diagnostic process can be a real challenge.

Case presentation: A 13-year female of Arab-Berber descent presented with abdominal pain and fever after a vacation in north-western Morocco. Complete blood count revealed a pancytopenia and blood smear revealed spirochetes. Borrelia hispanica was identified by sequencing the 16S rRNA gene. The patient was treated with tetracyclines and during this treatment we saw full clinical and hematological recovery.

Conclusions: Borrelia hispanica is a known cause of Tick-borne relapsing fever and is transmitted to humans through the bite of soft ticks of the genus Ornithodoros (Alectorobius). Although the link between tick-borne relapsing fever (TBRF) and thrombocytopenia has been documented, there are only a few case reports of TBRF presenting with pancytopenia. To the best of our knowledge, there is no previously report of Borrelia hispanica presenting with pancytopenia.
\end{abstract}

Keywords: Borrelia hispanica, children, pancytopenia

\section{Introduction}

Climate change increased migrant influx and increasing numbers of intercontinental travelers will cause higher prevalence of relatively unknown parasitic diseases in Western Europe. It is therefore not uncommon that a child with a febrile illness of unknown etiology is admitted to the hospital. The unfamiliarity of the pathology makes the diagnostic process a real challenge, especially when the presentation is unusual for an uncommon disease, like the one we would like to present here.

\section{Case presentation}

A 13-year old female of Arab-Berber descent (Morocco) presented to the emergency department because of abdominal cramps and pain in the right and left iliac fossa for three days accompanied by vomiting and high fever. One week before, she had returned from a two-month visit in northwestern Morocco. During her stay in Morocco she was residing in the house of a family member. There was no contact with animals, no history of a tick bite and she did not visit any parks or forests. She swam in the Mediterranean Sea and in a fresh water swimming pool but she never went swimming in natural fresh water resources. She had not been sick during her stay and no skin rash was seen. Returning to Belgium, the next day she developed lower abdominal pain, diarrhea, vomiting and high fever. After four days, she presented to the emergency department of the hospital.
Her medical history revealed a right-sided Bell's palsy in the previous year with an MRI that showed a neuritis facialis and a Borrelia serology that was negative. She had recurrent episodes of herpes labialis.

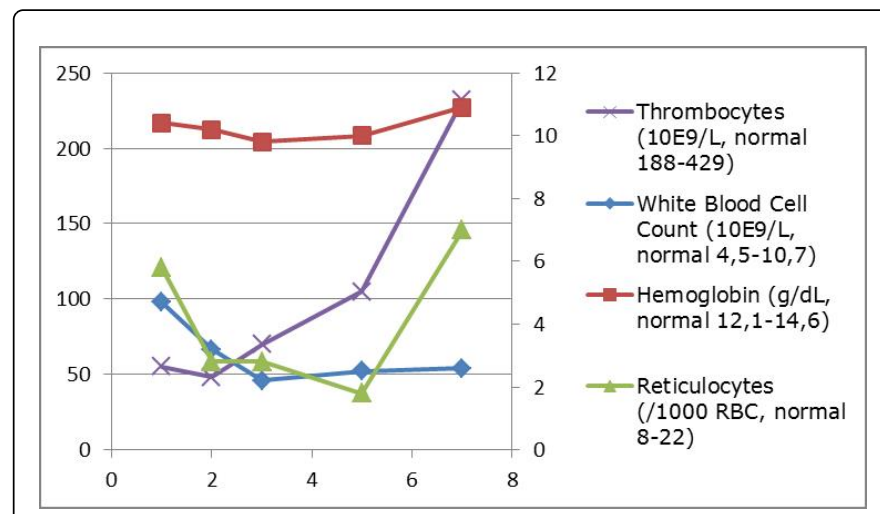

Figure 1: Blood count at diagnosis and response to therapy. Left yaxis: Thrombocytes. Right y-axis: White Blood cell Count, Hemoglobin and Reticulocytes. X-axis: time in days.

Physical examination at presentation showed a mildly sick, female with stable cardiovascular and respiratory parameters, without fever. She had a herpes labialis lesion on her lower lip. Heart- and lung auscultation were normal. The abdominal investigation showed normal bowel sounds and diffuse pain by palpation, most pronounced 
in right and left fossa. The initial complete blood count showed a mild, Coombs negative, normocytic anemia with a hemoglobin of $10,4 \mathrm{~g} / \mathrm{dL}$ (normal 12.1-14.6 g/dL), leukocytes of 4,7x10E9/L (normal $4.5-10.7 \times 10 \mathrm{E} 9 / \mathrm{L})$ with a mild neutropenia and mild monocytosis, and mildly enlarged thrombocytes of $55 \times 10 \mathrm{E} 9 / \mathrm{L}$ (normal $188-429 x 10 \mathrm{E} 9 / \mathrm{L}$ ), with C-reactive protein of $210 \mathrm{mg} / \mathrm{L}$ (normal $\leq 5$ $\mathrm{mg} / \mathrm{L}$ ). No signs of hemolysis with normal lactate dehydrogenase and bilirubin, and low reticulocyte count. Abdominal ultrasound was normal, but without visualization of the appendix. Urine investigation and thoracic X-ray showed no signs of infection. Since appendicitis could not be ruled out, an appendectomy was done immediately, however the appendix was not inflamed on visualization. The next day, the complete blood count revealed a more pronounced pancytopenia (see Figure 1) and the blood smear showed some spirochetes, so differential diagnosis was narrowed to leptospirosis or borreliosis (see Figure 2) making the latter more likely given the microscopic and morphological characteristics of the spirochetes. Serology for leptospirosis (IgM and IgG) and Borrelia burgdorferi were negative, as well as for ehrlichiosis, anaplasmosis, babesiosis, Rocky Mountain spotted fever and other parasitic infections. The diagnosis of tickborne relapsing fever was suspected and treatment with Tetracycline (IV, $20-40 \mathrm{mg} / \mathrm{kg} /$ day in 4 doses) was initiated.

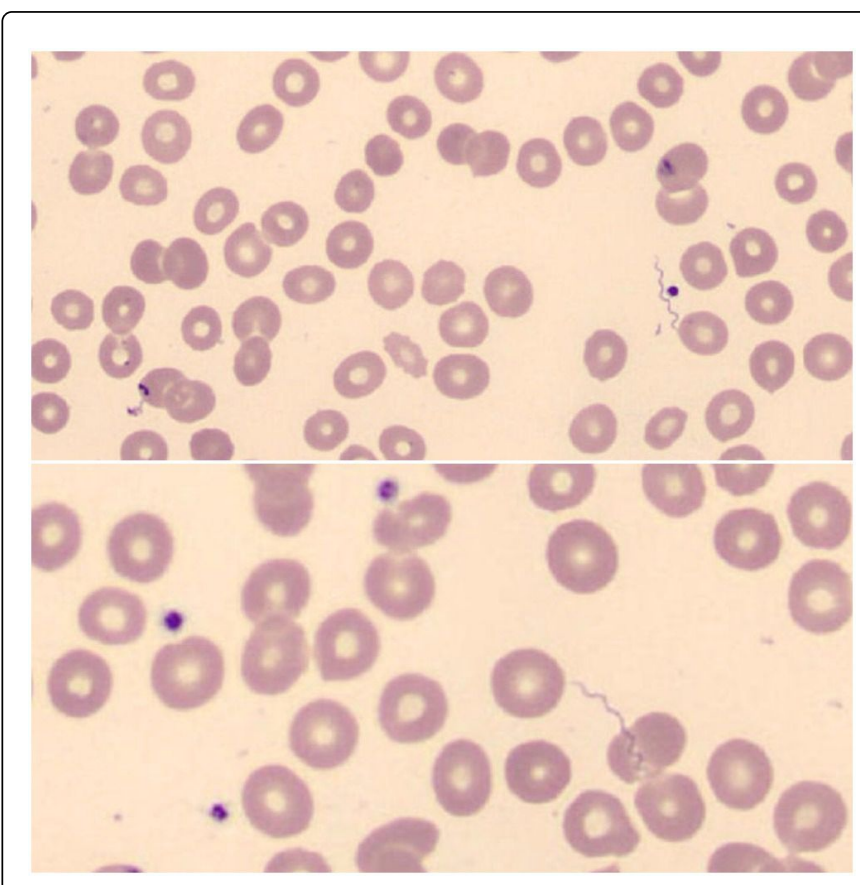

Figure 2: Blood smear revealing spirochetes with irregular, wide, open coils suggesting a Borrelia infection.

Blood- and urine cultures didn't reveal growth. Sequencing of $1500 \mathrm{bp}$ of the 16S rRNA gene (on a Genetic Analyzer ABI 3730XL (Applied Biosystems) with the BigDye Terminator kit (Applied Biosystems) using a home-brew method) confirmed our diagnosis and identified Borrelia hispanica. Our patient was treated with a combination of Ceftriaxone ( 2 gram/day in 1 dose, 3 days) and Doxycycline ( $2 \mathrm{mg} / \mathrm{kg} /$ day in 1 dose, 14 days) during which we saw full clinical and hematological recovery.

\section{Discussion}

Spirochetes are gram-negative bacteria with a double membrane and a helicoidal structure. Flagella are present allowing the spirochete to rotate as it moves. They are divided into three families (Leptospiraceae, Brachyspiraceae and Spirochaetaceae) and are responsible for several diseases in humans, for example leptospirosis, Lyme disease, relapsing fever, syphilis and intestinal spirochaetosis. Tick-borne relapsing fever (TBRF) is an infection caused by spirochetes of the genus Borrelia, transmitted to humans through the bite of soft ticks (Ornithodoros species). It is caused by at least 16 distinct Borrelia species throughout the world $[1,2]$. Borrelia spp. are gram-negative helical bacteria that normally measure 0.2 to $0.5 \mu \mathrm{m}$ in width and 5 to $20 \mu \mathrm{m}$ in length. They are very hard to culture but visible with dark-field and light microscopy. They have the corkscrew shape typical of all spirochetes [3]. Each Borrelia species associated with relapsing fever appears to be specific to its tick vector [2].

First signs of TBRF are usually observed between 4 and 14 days after the tick bite, with an acute onset of high fever, headache, arthralgia, myalgia, neck stiffness, and abdominal complaints [4]. Case fatality rate is $2-5 \%$ without treatment. Severity depends on Borrelia species, inoculum density, and underlying medical condition. Children and women appear to have a more intense course of disease [5]. The primary episode usually lasts 3 days and is followed, after a fever-free interval of 7 days, by multiple other alternating episodes, often shorter and milder. During the febrile periods, numerous Borreliae are circulating in the blood and diagnosis can be made by observation of spirochetemia on thin- or thick-blood smears with dark-field microscopy or with conventional microscopy after Giemsa, Wright or Diff-Quick staining $[5,6]$.

Borrelia cultures have not been widely used due to the low sensitivities range. Molecular methods are used with increasing frequency and offer the possibility of genus and even species identification [7]. As such, Borrelia hispanica has been detected and isolated from specimens obtained in Northern Africa and Southern Europe, including Morocco, Spain, Portugal, Greece and Cyprus and is held responsible for $20.5 \%$ of patients with unexplained fever in north western Morocco $[1,8]$. The disease caused by Borrelia hispanica is one of the less severe TBRFs, with neurological signs in less than $5 \%$ of cases [9]. The preferred treatment for TBRF is tetracycline or doxycycline. When contraindicated, erythromycin is the alternative. In very sick patients (with neurologic symptoms) IV Ceftriaxone can be added. No exact data are available in the literature about the association of TBRF and thrombocytopenia at presentation, but it is not uncommon [4]. In contrast, TBRF presenting with pancytopenia is rare and only reported in a few case reports. Two authors described infection with the recently discovered Borrelia miyamotoi and pancytopenia and there is also a case of a 59-old female with borreliosis and pancytopenia where the bone marrow was packed with Borrelia hermsii $[3,10]$. Pancytopenia is a common manifestation of many tick-borne diseases; however the pathogenesis is poorly understood, possibly resulting from decreased marrow production, consumption due to widespread endothelial damage or due to immune-mediated destruction. Data on the prevalence of thrombopenia, neutropenia or anemia with Borrelia hispanica TBRF are not available and to our knowledge, no reports of Borrelia hispanica presenting with pancytopenia have been published. Borrelia hispanica is transmitted through soft ticks, making co-infection of other tick-borne bacterial disease like anaplasmosis or babesiosis 
Citation: Leen I, Bruynseels P, Oort MV, Akker MVD (2016) A 13-Year Old Female with Pancytopenia at the Presentation of a Borrelia Hispanica Infection: Case Report and Review of the Literature. J Clin Case Rep 6: 871. doi:10.4172/2165-7920.1000871

Page 3 of 3

which are known to present with cytopenias, but transmitted through hard ticks of the Ixodes species, unlikely.

\section{Conclusion}

We report a case of Borrelia hispanica tick-borne relapsing fever presenting with abdominal pain, fever and pancytopenia. Tick borne relapsing fever is a rare disease in Europe, although found in some Meditterean countries, but is a frequent cause of fever in the NorthWestern part of Morocco. Therefore, it should be in the differential diagnosis of travelers returning from these areas presenting with unexplained fever. Although the link between tick-borne relapsing fever and thrombocytopenia has been documented, there are only a few cases of TBRF presenting with pancytopenia and, to the best of our knowledge, there is no previously report of Borrelia hispanica presenting with pancytopenia.

\section{References}

1. Rebaudet S, Parola P (2006) Epidemiology of relapsing fever borreliosis in Europe. FEMS Immunol Med Microbiol 48: 11-15.

2. Brouqui P, Bacellar F, Baranton G, Birtles RJ, Bjo ersdorff A, et al. (2004) Guidelines for the diagnosis of tick-borne bacterial diseases in Europe. Clin Microbiol Infect 10: 1108-1132.
3. Badger MS (2008) Tick talk: unusually severe case of tick-borne relapsing fever with acute respiratory distress syndrome-case report and review of the literature. Wilderness Environ Med 19:280-286.

4. Dworkin MS, Schwan TG, Anderson DE Jr, Borchardt SM (2008) Tickborne relapsing fever. Infect Dis Clin North Am 22: 449-468.

5. Barbour AG (1999) Relapsing fever and other Borrelia infections. Tropical Infectious Diseases: Principles, Pathogens, and Practice.

6. Johnson WD GL (2000) Borrelia species (relapsing fever). Mandell, Douglas, and Bennett's Principles and Practice of Infectious Diseases. New York: Churchill Livingstone, USA.

7. Brahim H, Perrier-Gros-Claude JD, Postic D, Baranton G, Jambou R (2005) Identifying relapsing fever Borrelia, Senegal. Emerg Infect Dis 11: 474-475.

8. Sarih M, Garnier M, Boudebouch N, Bouattour A, Rihani A, et al. (2009) Borrelia hispanica relapsing fever, Morocco. Emerg Infect Dis 15: 1626-1629.

9. Cadavid D, Barbour AG (1998) Neuroborreliosis during relapsing fever: review of the clinical manifestations, pathology, and treatment of infections in humans and experimental animals. Clin Infect Dis 26: 151-164.

10. Chowdri HR, Gugliotta JL, Berardi VP, Goethert HK, Molloy PJ, et al. (2013) Borrelia miyamotoi infection presenting as human granulocytic anaplasmosis: a case report. Ann Intern Med 159: 21-27. 